PROCEEDINGS OF THE

AMERICAN MATHEMATICAL SOCIETY

Volume 129, Number 8, Pages 2219-2225

S 0002-9939(00)05831-7

Article electronically published on December 28, 2000

\title{
SIGNS IN THE $c d$-INDEX OF EULERIAN PARTIALLY ORDERED SETS
}

\author{
MARGARET M. BAYER
}

(Communicated by John R. Stembridge)

\begin{abstract}
A graded partially ordered set is Eulerian if every interval has the same number of elements of even rank and of odd rank. Face lattices of convex polytopes are Eulerian. For Eulerian partially ordered sets, the flag vector can be encoded efficiently in the $c d$-index. The $c d$-index of a polytope has all positive entries. An important open problem is to give the broadest natural class of Eulerian posets having nonnegative $c d$-index. This paper completely determines which entries of the $c d$-index are nonnegative for all Eulerian posets. It also shows that there are no other lower or upper bounds on $c d$-coefficients (except for the coefficient of $c^{n}$ ).
\end{abstract}

\section{INTRODUCTION}

In the past thirty years or more, there has been much interest in combinatorial questions about polytopes and other geometric complexes and partial orders. Of central importance is the flag vector of a partially ordered set (poset) and various combinatorial parameters derived from it. One of these parameters is the $c d$-index, defined for Eulerian posets, a class that contains face lattices of polytopes. The $c d$ index was discovered by Fine and introduced in the literature by Bayer and Klapper (4]). It has captured the imagination, both for what is known and for what is not known about it. It embodies in an elegant way the linear relations of flag vectors of Eulerian posets (the generalized Dehn-Sommerville relations of Bayer and Billera [1]); the number of coefficients in the $c d$-index is a Fibonacci number. It is known to be nonnegative for polytopes (see Stanley [12]), but it is not known what it counts, except in special cases (see Purtill [11). Among polytopes, the $c d$-index is minimized by the simplices (see Billera and Ehrenborg [5]). Novik ([10]) gives lower bounds for $c d$-coefficients of odd-dimensional simplicial manifolds (or, more generally, Eulerian Buchsbaum complexes).

Stanley ([12]) proved the nonnegativity of the $c d$-index for "S-shellable" regular CW-spheres (including polytopes). In [13] he proposes the following as the main open problem concerning the $c d$-index: Is the $c d$-index nonnegative for all Gorenstein* posets? (These are the Cohen-Macaulay Eulerian posets.) In fact, some parts of the $c d$-index are nonnegative for all Eulerian posets. In this paper we determine which $c d$-words have nonnegative coefficients for all Eulerian posets. For all other $c d$-words, we show how to construct Eulerian posets with arbitrarily

Received by the editors October 22, 1999 and, in revised form, December 21, 1999.

2000 Mathematics Subject Classification. Primary 06A07.

Key words and phrases. Eulerian poset, flag vector, $c d$-index. 
large negative coefficients. The proofs grow out of the ideas of [2, which studies the cone of flag vectors of Eulerian posets.

\section{Definitions}

An Eulerian poset is a graded partially ordered set $P$ in which every interval has the same number of elements of even and of odd rank. For $P$ an Eulerian poset, the dual poset, obtained by reversing the order relation, is also Eulerian. The $c d$-index of an Eulerian poset is an invariant based on the numbers of chains in the poset. For $P$ an Eulerian poset of rank $n+1$ and $S \subseteq[1, n], f_{S}(P)$ is the number of chains in $P$ of the form $\hat{0} \prec x_{1} \prec x_{2} \prec \cdots \prec x_{k} \prec \hat{1}$, where $\left\{\operatorname{rank}\left(x_{i}\right): 1 \leq i \leq k\right\}=S$. The $2^{n}$-tuple of flag numbers $f_{S}(P)$ (as $S$ ranges over all subsets of $[1, n]$ ) is called the flag vector of $P$. The flag h-vector is obtained by performing inclusion-exclusion on the flag vector. Thus $h_{S}=\sum_{T \subseteq S}(-1)^{|S \backslash T|} f_{T}$ or, equivalently, $f_{S}=\sum_{T \subseteq S} h_{T}$. Write a generating function in noncommuting variables, $\Psi(a, b)=\sum h_{S} u_{S}$, where $u_{S}=u_{1} u_{2} \cdots u_{n}$ with $u_{i}=a$ if $i \notin S$ and $u_{i}=b$ if $i \in S$. For every Eulerian poset, there is a polynomial $\Phi(c, d)$ in noncommuting variables $c$ and $d$ for which $\Psi(a, b)=\Phi(a+b, a b+b a)$. The polynomial $\Phi(c, d)$ (or $\Phi_{P}(c, d)$ when we need to specify the poset $P$ ) is called the $c d$-index of the poset. The $c d$-index of the dual of the Eulerian poset $P$ is obtained by reversing every $c d$-word in the $c d$-index of $P$. The coefficient of a $c d$-word $w$ is written as $[w]\left(\right.$ or $\left.[w]_{P}\right)$. We think of each $d$ as occupying two positions in a $c d$-word, namely, the positions of $a b$ or $b a$ in the corresponding $a b$-words. Let $\operatorname{supp}(w)$ be the set of positions of $d$ in $w$.

Stanley ([12]) notes a useful variation of the $c d$-index. The $c e$-index is obtained by replacing every $d$ in $\Phi(c, d)$ by $(c c-e e) / 2$. Alternatively, one gets the $c e$-index from $\Psi(a, b)$ - even for non-Eulerian posets - by letting $c=a+b$ and $e=a-b$. The $c e$-index is thus a polynomial in the noncommuting variables $c$ and $e$, where for Eulerian posets the $e$ 's occur only in pairs. Write $L_{Q}$ for the coefficient of the word $v_{Q}=v_{1} v_{2} \cdots v_{n}$, where $v_{i}=c$ if $i \notin Q$ and $v_{i}=e$ if $i \in Q$. The vector of coefficients of the ce-index, $\left(L_{Q}(P)\right)$, is also known as the $L$-vector of $P$.

For an Eulerian poset $P, L_{Q}(P)=0$ unless $Q$ is an even set, that is, $Q$ is the union of disjoint intervals of even cardinality. We say $Q$ evenly contains $S$, written $S \subseteq e Q$, if $S$ and $Q$ are even sets, $S \subseteq Q$, and the difference set $Q \backslash S$ is also an even set. An "Eulerian" $c e$-word $v_{Q}$ is converted to a sum of $c d$-words by replacing consecutive pairs of $e$ 's in $v_{Q}$ by $c c-2 d$ so that no $e$ 's remain. This means that a $c d$-word $w$ occurs in the expansion of a $c e$-word $v_{Q}$ if and only if $\operatorname{supp}(w) \subseteq_{e} Q$. Thus the coefficient in the $c d$-index of a $c d$-word $w$ in which $d$ occurs $r$ times is

$$
[w]=(-2)^{r} \sum_{\operatorname{supp}(w) \subseteq_{e} Q} L_{Q} .
$$

(See [2] for more information on $L$-vectors.)

In determining the cone of flag vectors of all graded posets ([6]), Billera and Hetyei construct sequences of posets with convergent (normalized) flag vectors. Bayer and Hetyei apply a doubling operation to some of these to get sequences of Eulerian posets. Given an interval $I=[i, j] \subseteq[1, n]$, a rank $n+1$ poset $P$ and a positive integer $N$, let $D_{I}^{N}(P)$ be the rank $n+1$ poset obtained by replacing $P_{I}$, the subposet of $P$ consisting of elements with ranks in $I$, by $N$ copies of itself. The (horizontal) double DP of a poset $P$ is the result of starting with $P$ and successively applying the operators $D_{\{i\}}^{2}$, for $1 \leq i \leq n$. (In the Hasse diagram of $P$ every edge 
is replaced by $\bowtie$.) For $\mathcal{I}$ a set of subintervals of $[1, n], \mathcal{I}$ is an even interval system if (1) no interval of $\mathcal{I}$ is contained in another, (2) every interval of $\mathcal{I}$ is of even cardinality, and (3) the intersection of any two intervals of $\mathcal{I}$ is of even cardinality. For each even interval system $\mathcal{I}$ over $[1, n]$, there exists a sequence of Eulerian posets, $D P(n, \mathcal{I}, N)$, whose normalized flag vectors (and hence, normalized $c d$ indices and $c e$-indices) converge. These are obtained by starting with a rank $n+1$ chain, successively applying the operators $D_{I}^{N}$ for the intervals $I \in \mathcal{I}$, and finally taking the horizontal double.

For $\mathcal{I}$ an interval system of $k$ intervals, write

$$
L_{S}(D P(n, \mathcal{I}))=\lim _{N \rightarrow \infty} L_{S}(D P(n, \mathcal{I}, N)) / N^{k}
$$

(Here $2^{n} N^{k}$ is the number of maximal chains in $D P(n, \mathcal{I}, N)$.) The symbol $D P(n, \mathcal{I})$ is referred to as a limit poset. These ce-index coefficients are given by the formula

$$
L_{S}(D P(n, \mathcal{I}))=\sum_{j=0}^{k}(-1)^{j}\left|\left\{1 \leq i_{1}<\cdots<i_{j} \leq k: I_{i_{1}} \cup \cdots \cup I_{i_{j}}=S\right\}\right|,
$$

where $\mathcal{I}=\left\{I_{1}, I_{2}, \ldots, I_{k}\right\}$. See [2] for details. The formula applies for non-Eulerian limit posets as well; in that case it can give nonzero $L_{Q}$ for noneven sets $Q$.

We use one other result stated and proved in [2] (but implicit in [7]).

Proposition (Inequality Lemma). Let $T$ and $V$ be subsets of $[1, n]$ with $T \subseteq V$, such that for every maximal interval $I$ of $V,|I \cap T| \leq 1$. Write $S=[1, n] \backslash V$. For $P$ any rank $n+1$ Eulerian poset,

$$
\sum_{R \subseteq T}(-2)^{|T \backslash R|} f_{S \cup R}(P) \geq 0 .
$$

Equivalently,

$$
(-1)^{|T|} \sum_{T \subseteq Q \subseteq V} L_{Q}(P) \geq 0
$$

\section{The MAIN RESUlt}

Theorem. 1. For the following cd-words $w$, the coefficient of $w$ as a function of Eulerian posets has greatest lower bound 0 and has no upper bound:

(a) $c^{i} d c^{j}$, with $\min \{i, j\} \leq 1$,

(b) $c^{i} d c d \cdots c d c^{j}$ (at least two d's alternating with $c$ 's, $i$ and $j$ unrestricted).

2. The coefficient of $c^{n}$ in the cd-index of every Eulerian poset $i s 1$.

3. For all other $c d$-words $w$, the coefficient of $w$ as a function of Eulerian posets has neither lower nor upper bound.

Note. For $n \geq 5$, there are $\left\lfloor\left(\begin{array}{c}n-2 \\ 2\end{array}\right) / 3\right\rfloor+4 c d$-words of the types described in part 1. This is a small portion of the $c d$ words for large $n$.

Proof. The fact that the coefficient of $c^{n}$ is 1 is immediate from the definition and is included only for completeness.

Let $w$ be any $c d$-word containing $r$ copies of $d$, with $r \geq 1$. Let $\mathcal{I}$ be the set of two-element intervals of the positions of $d$ in $w$. Compute the coefficient of $w$ in the $c d$-index of $D P(n, \mathcal{I})$. If $Q$ properly contains $\operatorname{supp}(w)$, then by equation (2), $L_{Q}(D P(n, \mathcal{I}))=0$. So by equations (11) and (2), for $D P(n, \mathcal{I})$, the coefficient of $w$ is $[w]=(-2)^{r} L_{\operatorname{Supp}(w)}(D P(N, \mathcal{I}))=(-2)^{r}(-1)^{r}=2^{r}$. This is the limit as $N$ goes 
to infinity of $1 / N^{r}$ times $[w]$ for $D P(n, \mathcal{I}, N)$. So $(D P(n, \mathcal{I}, N))$ is a sequence of Eulerian posets with $c d$-coefficients $[w]$ not bounded above. (Earlier Stanley ([12]) showed this using a different sequence of posets.)

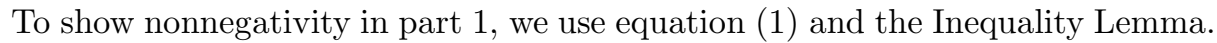
If $w=d c^{j}$, let $S=\emptyset$ (so $V=[1, n]$ ) and $T=\{1\}$. Then the coefficient of $w$ is $[w]=2(-1) \sum_{T \subseteq Q \subseteq V} L_{Q} \geq 0$. If $w=c d c^{j}$ let $S=\{1\}$ (so $\left.V=[2, n]\right)$ and $T=\{2\}$. Then the coefficient of $w$ is $[w]=(-2) \sum_{\{2,3\} \subseteq_{e} Q} L_{Q}=2(-1) \sum_{T \subseteq Q \subseteq V} L_{Q} \geq 0$, because $L_{Q}$ is zero unless $Q$ is an even set. The cases of $w=c^{i} d$ and $w=c^{i} d c$ follow by duality.

Let $w=c^{i} d c d \cdots c d c^{j}$, with $d$ occurring $r$ times, $r \geq 2$; thus

$$
\operatorname{supp}(w)=\{i+1, i+2, i+4, i+5, \ldots, i+3 r-2, i+3 r-1\} .
$$

Let

$$
\begin{gathered}
S=\{i+3, i+6, \ldots, i+3 r-3\}, \\
V=[1, n] \backslash S
\end{gathered}
$$

and

$$
T=\{i+2, i+4, i+7, \ldots, i+3 r-5, i+3 r-2\} .
$$

Here $S$ is the set of positions of the $c$ 's between $d$ 's and $T$ is a set of one position for each $d$, adjacent to the positions of the interior $c$ 's. The coefficient of $w$ is $[w]=(-2)^{r} \sum_{\operatorname{supp}(w) \subseteq_{e} Q} L_{Q}$. The set $Q$ evenly contains $\operatorname{supp}(w)$ if and only if $Q$ is an even set and $T \subseteq Q \subseteq V$. Since $L_{Q}=0$ unless $Q$ is an even set, $[w]=2^{r}(-1)^{r} \sum_{T \subseteq Q \subseteq V} L_{Q} \geq 0$.

The double of the chain, $D C^{n+1}$, has $c d$-index $c^{n}$, so for the $c d$-coefficients in part 1 , the lower bound of 0 is actually attained.

It remains to show that the coefficients of the $c d$ words in part 3 can be arbitrarily negative. We use several lemmas.

Lemma 1. For every even $n \geq 4$ the coefficient of $d c^{n-4} d$ as a function of Eulerian posets has no lower bound.

Proof. Let $\mathcal{I}=\{[1, n]\}$. By equation (2) the only nonzero entries in the $L$-vector of $D P(n, \mathcal{I})$ are $L_{\emptyset}=1$ and $L_{[1, n]}=-1$. By (11) the coefficient of $d c^{n-4} d$ in the $c d$-index of $D P(n, \mathcal{I})$ is $(-2)^{2}(-1)=-4$. This is the limit as $N$ goes to infinity of $1 / N^{2}$ times $\left[d c^{n-4} d\right]$ for $D P(n, \mathcal{I}, N)$. So $(D P(n, \mathcal{I}, N))$ is a sequence of Eulerian posets with $c d$-coefficients $\left[d c^{n-4} d\right]$ not bounded below. (A formula of Ehrenborg and Readdy ([8]) gives directly that the $c d$-index of $D P(n, \mathcal{I}, N)$ is $\left.(N+1) c^{n}-N(c c-2 d)^{n / 2}.\right)$

In [3] Bayer and Hetyei discuss constructions of Eulerian posets whose normalized $L$-vectors converge to sums of $L$-vectors of non-Eulerian Billera-Hetyei limit posets. (A few examples are found in [2, Appendix A].)

Lemma 2. For every odd $n \geq 7$ the coefficient of $d c^{n-4} d$ as a function of Eulerian posets has no lower bound.

Proof. Write $C^{n+1}$ for the chain of rank $n+1$. Let

$$
P^{I}(N)=D_{[1,2]}^{N+1} D_{[3, n-3]}^{N+1} D_{[4, n-2]}^{N+1} D_{[n-1, n]}^{N+1}\left(C^{n+1}\right) ;
$$


let

$$
P^{I I}(N)=D_{[1, n-3]}^{N+1} D_{[3, n-2]}^{N^{2}} D_{[4, n]}^{N+1}\left(C^{n+1}\right)
$$

and let

$$
P^{I I I}(N)=D_{[1, n]}^{N^{4}}\left(C^{n+1}\right) .
$$

Create a poset $P(N)$ from these three posets by identifying the elements of $P^{I I}(N)$ with the elements of $P^{I}(N)$ at ranks $0,1,2, n-1, n$, and $n+1$, and then identifying the elements of $P^{I I I}(N)$ with the elements of $P^{I}(N)$ and $P^{I I}(N)$ only at ranks 0 and $n+1$. The doubles $D P(N)$ of these posets are Eulerian, and the normalized $L$-vectors converge as $N$ goes to infinity. Write $L_{Q}(D P)=$ $\lim _{N \rightarrow \infty} L_{Q}(D P(N)) / f_{[1, n]}(D P(N))$. Then

$$
L_{Q}(D P)=L_{Q}\left(D P\left(n, \mathcal{I}_{1}\right)\right)+L_{Q}\left(D P\left(n, \mathcal{I}_{2}\right)\right)+L_{Q}\left(D P\left(n, \mathcal{I}_{3}\right)\right),
$$

where $\mathcal{I}_{1}=\{[1,2],[3, n-3],[4, n-2],[n-1, n]\}, \mathcal{I}_{2}=\{[1, n-3],[3, n-2],[4, n]\}$, and $\mathcal{I}_{3}=\{[1, n]\}$. The only nonzero $L_{Q}$ for which $\{1,2, n-1, n\} \subseteq_{e} Q$ are $L_{\{1,2, n-1, n\}}(D P)=1, L_{[1, n] \backslash\{3\}}(D P)=-1$ and $L_{[1, n] \backslash\{n-2\}}(D P)=-1$, so by equation (11) the coefficient of $d c^{n-4} d$ in the $c d$-index of $D P$ is -4 . This is the limit as $N$ goes to infinity of $1 / f_{[1, n]}(D P(N))$ times $\left[d c^{n-4} d\right]$ for $D P(N)$. So $(D P(N))$ is a sequence of Eulerian posets with $c d$-coefficients $\left[d c^{n-4} d\right]$ not bounded below. (In fact, a flag vector calculation gives the coefficient of $d c^{n-4} d$ for $D P(N)$ as $4\left(N^{2}-N^{4}\right)$.)

The proof of Lemma 2 asserts that $D P(N)$ is Eulerian. It is easy to check by equation (21) that $L_{Q}\left(D P\left(n, \mathcal{I}_{1}\right)\right)+L_{Q}\left(D P\left(n, \mathcal{I}_{2}\right)\right)+L_{Q}\left(D P\left(n, \mathcal{I}_{3}\right)\right)=0$ if $Q$ is not an even set. This condition must hold if every $D P(N)$ is an Eulerian poset. But to prove that $D P(N)$ is Eulerian requires us to show that every interval of the poset has the same number of elements of even rank and of odd rank. We show the details in one case. Let $[x, y]$ be an interval of $P(N)$ with $x$ of rank 2 and $y$ of rank $n-1$. For the Eulerian condition to hold on corresponding intervals in $D P(N)$, the interval $[x, y]$ of $P(N)$ must have one more element of even rank than of odd rank. If $x$ and $y$ are in the subposet $P^{I I I}(N)$, then $[x, y]$ has exactly one element of each rank, so the condition is met. Suppose $x$ and $y$ are identified elements of $P^{I}(N)$ and $P^{I I}(N)$. In the open interval $(x, y)$ in $P^{I}(N)$, ranks 3 and $n-2$ each have $N+1$ elements and each other rank has $(N+1)^{2}$ elements. In the open interval $(x, y)$ in $P^{I I}(N)$, each rank has $N^{2}$ elements. So the number of even-rank elements in $[x, y]$ is $2+\left((N+1)^{2}+N^{2}\right)(n-5) / 2$, and the number of odd-rank elements in $[x, y]$ is $2(N+1)+2 N^{2}+\left((N+1)^{2}+N^{2}\right)(n-7) / 2$. The difference is 1 . Note that neither $P^{I}(N)$ nor $P^{I I}(N)$ satisfies the Eulerian condition for $[x, y]$ by itself. The two subposets balance each other to achieve the Eulerian property. This works for all intervals.

Lemma 3. The coefficient of ccdcc as a function of rank 7 Eulerian posets has no lower bound.

Proof. The following limit poset is given in Appendix A of [2]. Let $P^{I}(N)=$ $D_{[1,2]}^{N} D_{[2,6]}^{N}\left(C^{7}\right)$ and $P^{I I}(N)=D_{[1,5]}^{N} D_{[5,6]}^{N}\left(C^{7}\right)$. Let $P(N)$ be formed from these two posets by identifying the elements at ranks $0,1,6$, and 7 . The double $D P(N)$ of this poset is Eulerian. In the limit, the normalized $L$-vector includes the following 
values: $L_{34}=L_{1234}=L_{3456}=0$, and $L_{123456}=1$. These are the $L_{Q}$ that contribute to the coefficient of $c c d c c$ in the $c d$-index,

$$
[c c d c c]=-2\left(L_{34}+L_{1234}+L_{3456}+L_{123456}\right)=-2 .
$$

As argued before, this gives a sequence of Eulerian posets with $c d$-coefficients [ccdcc] not bounded below. (In fact, for $D P(N),[c c d c c]=-2(N-1)^{2}$.)

Lemma 4. Let $u$ and $v$ be cd-words. If the coefficient of $u$ as a function of Eulerian posets has no lower bound, then the coefficients of uv and vu as functions of Eulerian posets have no lower bounds.

Proof. In 12 Stanley considers a "join" operation, which produces an Eulerian poset $P * Q$ from two Eulerian posets $P$ and $Q$. He shows that the $c d$-indices satisfy $\Phi_{P * Q}(c, d)=\Phi_{P}(c, d) \Phi_{Q}(c, d)$. Let $u$ be a $c d$-word of length $m$ and $v$ a $c d$-word of length $n$. Let $B$ be the rank $n+1$ Boolean algebra. Every $c d$-word of length $n$ has a positive coefficient in the $c d$-index of $B$. (This is proved most easily from the Ehrenborg-Readdy formula for the $c d$-index of a pyramid in [8].) Let $P_{N}$ be a sequence of rank $m+1$ Eulerian posets for which $\lim _{N \rightarrow \infty}[u]_{P_{N}}=-\infty$. Then $\lim _{N \rightarrow \infty}[u v]_{P_{N} * B}=\lim _{N \rightarrow \infty}[v u]_{B * P_{N}}=-\infty$.

We now complete the proof of the theorem. Every $c d$-word not included in parts 1 and 2 of the Theorem contains the subword $c c d c c$ or a subword of the form $d c^{n-4} d$ for $n-4 \neq 1$. Thus, by Lemmas 1 through 4 , the coefficients of these $c d$-words as functions of Eulerian posets have no lower bounds.

\section{ACKNOWLEDGMENTS}

The referee was most generous with suggestions for improving the paper. I also wish to thank Gábor Hetyei for introducing me to the construction of Eulerian posets crucial to this paper, and for other helpful discussions.

\section{REFERENCES}

[1] Margaret M. Bayer and Louis J. Billera, Generalized Dehn-Sommerville relations for polytopes, spheres and Eulerian partially ordered sets, Invent. Math. 79 (1985), 143-157. MR 86f:52010b

[2] Margaret M. Bayer and Gábor Hetyei, Flag vectors of Eulerian partially ordered sets, European J. Combin. (to appear).

[3] , Generalizations of Eulerian partially ordered sets, flag numbers, and the Möbius function, preprint 2000.

[4] Margaret M. Bayer and Andrew Klapper, A new index for polytopes, Discrete Comput. Geom. 6 (1991), 33-47. MR 91k:52024

[5] Louis J. Billera and Richard Ehrenborg, Monotonicity of the cd-index for polytopes, Math. Z. 233 (2000), 421-441.

[6] Louis J. Billera and Gábor Hetyei, Linear inequalities for flags in graded partially ordered sets, J. Combin. Theory Ser. A 89 (2000), 77-104. CMP 2000:07

[7] Louis J. Billera and Niandong Liu, Noncommutative enumeration in graded posets, J. Algebraic Combin. 12 (2000), 7-24.

[8] Richard Ehrenborg and Margaret Readdy, Coproducts and the cd-index, J. Algebraic Combin. 8 (1998), 273-299. MR 2000b:52009

[9] Gil Kalai, A new basis of polytopes, J. Combin. Theory Ser. A 49 (1988), 191-209. MR 90a:52012

[10] Isabella Novik, Lower bounds for the cd-index of odd-dimensional simplicial manifolds, European J. Combin. 21 (2000), 533-541.

[11] Mark Purtill, André permutations, lexicographic shellability and the cd-index of a convex polytope, Trans. Amer. Math. Soc. 338 (1993), 77-104. MR 93j:52017 
[12] Richard P. Stanley, Flag f-vectors and the cd-index, Math. Z. 216 (1994), 483-499. MR 96b:06006

[13] — Positivity problems and conjectures in algebraic combinatorics, Mathematics: Frontiers and Perspectives, Amer. Math. Soc., Providence, RI, 2000, pp. 295-319. CMP 2000:13

Department of Mathematics, University of Kansas, Lawrence, Kansas 66045-2142

E-mail address: bayer@math.ukans.edu 